\title{
THE STUDY ON GATA4 GENE MUTATIONSIN CHINESE PATIENTS WITH CONGENITAL
} HEART DISEASE

\author{
J.D. Ding, K. Li, X. Fang, X. Zhang, Y. Yao, G. Ma \\ Department of Cardiology, Zhongda Hospital \& School of Clinical Medicine Southeast University, Nanjing, \\ China
}

Background: Congenital heart diseases (CHD) are the most common heart developmental anomaly disease. It occurs in approximately $0.4 \% \sim 5 \%$ of all newborns and continues to be a significant cause of death in infancy. Nevertheless, the aetiology of CHD in the majority of cases remains unknown. Heart formation is a complex process regulated by many transcription factors. Although most of CHD cases are sporadic, there are several genes which were found to be associated with CHD, including NKX2.5, TBX5, GATA4, etc.

Objective: The study aimed to identify the mutations of GATA4 gene in Chinese patients with sporadic CHD.

Methods: 99 patients with CHD were recruited in this study, among which 6 patients had a variety of complex heart defects and 93 patients had simple heart defects, such as atrial septal defect (ASD), ventricular septal defect (VSD), pulmonary valvar stenosis (PVS) and patent ductus arteriosus (PDA). 90 normal subjects were selected as control. After amplifying the exon 3 of the GATA4 gene by polymerase chain reaction (PCR), we purified the PCR products and conducted the sequencing reaction, analyzed the mutation of the GATA4 gene.

Results: Three inserted mutations $(52,58,61)$ with base A in the exon 3 of the GATA4 gene were identified in 3 of 21 patients with PDA.

Conclusion: Our results suggest that GATA4 gene mutations may be one of etiologic causes of congenital heart disease in Chinese people.

Keywords: GATA4; congenital heart disease; gene mutation 